\title{
Effet du travail du sol sur la productivité de différentes provenances de Jatropha curcas
}

\author{
Lamine BARRO ${ }^{1}$ \\ Ndiaye Arona Samba SAMBA ${ }^{2}$ \\ Malaïny DIATTA ${ }^{3}$ \\ Elie Léonard $\mathrm{AKPO}^{1}$ \\ 1 Université Cheikh Anta Diop de Dakar, \\ BP 5005, \\ Dakar, \\ Sénégal \\ 2 Université de Thiès, \\ Ecole Nationale Supérieure d'Agriculture, \\ BP 967 Thies, \\ Sénégal \\ <bathie_samba@yahoo.fr> \\ 3 Institut Sénégalais de Recherches \\ Agricoles/CNRF, \\ BP 2312, \\ Dakar, \\ Sénégal
}

Article reçu le 11 novembre 2011

Accepté le 30 janvier 2013

Jatropha curcas L. (JCL ou pourghère), originaire d'une zone couvrant le Mexique, I'Amérique centrale, le Brésil, la Bolivie, le Pérou, I'Argentine et le Paraguay, est un arbuste de 5-7 m qui appartient à la famille des Euphorbiaceae (USDA ANGRPNGRLBM, 2007). Il a été introduit dans de nombreuses régions subtropicales et tropicales où il est utilisé comme haie vive ou comme plante médicinale (Heller, 1996). Son aire de distribution actuelle couvre les zones arides et semi-arides (Jones et Miller, 1992 ; Makkar et Becker, 1997) et les régions tropicales humides : Guatemala (> $4000 \mathrm{~mm} / \mathrm{an})$, nord du Vietnam et de la Thaïlande. Rijssenbeek et al. (2007) situent son aire entre les latitudes $30^{\circ} \mathrm{N}$ et $35^{\circ} \mathrm{S}$. JCL est résistant à la sécheresse mais exige des sols bien drainés,

\begin{abstract}
Effect of soil preparation and plant material provenances on Jatropha curcas productivity

Jatropha curcas L. (JCL), an oleaginous species traditionally used as live fence, is massively planted in Africa to produce biofuel. However, the influence of many factors on its productivity remains scientifically unknown. This study aimed to assess the effect of subsoiling (factor 1: subsoiling and control) and plant material provenance (factor 2: Casamance and Diobass) on its development and productivity. The results have shown two years after planting that plant provenance had a significant effect on growth variables, plant above ground architecture and fruits number. The Casamance provenance has generally produced the highest values. Subsoiling did not have a significant effect on most of the studied variables, except for low primary branches number and fruiting branches number. Even more, subsoiling appeared to enhance JCL growth. These results have allowed to understand the importance of provenances but also of soil preparation on the performances of JCL and to identify priority areas for research.
\end{abstract}

Key words: Jatropha curcas, productivity, provenance, ramification, soil preparation

notamment dans les zones à plus forte pluviométrie (Legendre et al., 2009).

L'arbre peut jouer un rôle dans la lutte contre l'érosion. II est utilisé en médecine traditionnelle : I'huile a des propriétés purgatives à petite dose et sert à traiter certaines maladies de la peau ou à soulager les rhumatismes. JCL a d'autres utilisations comme savon artisanal ou comme moyen d'éclairage avec des lampes rudimentaires. Le latex, utilisé sur les blessures, aurait des propriétés antiseptiques et coagulantes (Dommergue Pirot, 2008).

Des extraits aqueux de pourghère peuvent être utilisés comme biocontrôle $d$ 'agents pathogènes fongiques et exercent une activité molluscicide contre la douve du foie (Emesto, 2008). Les autres valorisations sont I'utilisation du tourteau des graines triturées comme engrais organique ou pour la production d'énergie (combustion, cogénération) (Dommergue et Pirot, 2008).

Parmi les différents choix de sources d'énergies renouvelables, les plantes oléagineuses comme JCL présentent un potentiel pour satisfaire une partie des besoins croissants en énergie, surtout dans les pays pauvres d'Afrique non producteurs de pétrole. Ses graines contiennent 30-40\% d'huile avec un acide gras similaire à celui des huiles comestibles (Gübitz et al., 1999). L'huile après filtration peut être utilisée dans différents types de moteurs de conception classique. Une estérification est cependant nécessaire pour son usage

Pour citer cet article : Barro L, Samba NSS, Diatta M, Akpo EL. Effet du travail du sol sur la productivité de différentes provenances de Jatropha curcas. OCL $2013 ; 20(3)$ : 165-170. doi : 10.1684/ocl.2013.0508 
dans les moteurs diesel modernes (Dommergue et Pirot, 2008).

En dépit de toutes les potentialités qu'on reconnaît à $\mathrm{JCL}$, force est de reconnaître que les connaissances scientifiques disponibles sur cette plante sont encore fragmentaires. Peu de données sont ainsi disponibles sur les différentes provenances de JCL et les effets du travail du sol sur leur développement et productivité en zone sahélienne. Toutefois, Makkar et Becker (1997) ont observé sur 18 provenances de JCL issues de différents pays d'Afrique, des Amériques et d'Asie $d^{\prime}$ importantes variations sur les teneurs en protéines et graisses brutes, en éléments pariétaux et en cendres, mais aucun lien de causalité n'a été observé. Kaushik et al. (2007) ont observé sur 24 provenances de JCL en Inde (Haryana) la prédominance du facteur environnemental sur le facteur génétique pour la taille des graines, leur poids et teneur en huile. Par ailleurs, la variabilité génétique observée entre les provenances de JCL en Inde est faible contrairement à celle qui existe entre les génotypes indiens et mexicains (Basha et Sujatha, 2007).

Cette présente étude avait pour objectif d'évaluer l'effet du sous-solage et de la provenance sur le développement (variables de croissances et architectures des ports aériens) des plants de JCL âgés de 24 mois et sur leur productivité (nombre de fruits et masse des graines/ arbre/an).

\section{Matériel et méthodes}

\section{Milieu d'étude}

La zone d'étude se situe à l'Est de la ville de Bargny sur le site de la SOCOCIM (14 $42^{\prime} 26^{\prime \prime} \mathrm{N}, 17^{\circ} 14^{\prime} 48^{\prime \prime} \mathrm{O}$ ). L'altitude moyenne est de $20 \mathrm{~m}$ avec une nappe phréatique profonde de 12 à 36 mètres (Diallo et al., 2006). Le climat, de type soudano-sahélien, est caractérisé par l'alternance d'une saison sèche longue de 9 mois (octobre à juin) et d'une saison des pluies de trois mois (juillet à septembre). Le site se trouve entre les isohyètes 200 et $500 \mathrm{~mm}$; l'année de plantation 2008 a enregistré 49 jours de pluies, répartis comme suit : 5 jours en juin, 9 en juillet, 16 en août, 17 en septembre et 2 en octobre pour un cumul pluviométrique annuel de 378 mm. L'influence de l'Alizé maritime adoucit les températures avec une moyenne annuelle de $24,2{ }^{\circ} \mathrm{C}$ alors que celle des températures maximales s'élève à $27,3^{\circ} \mathrm{C}$. La valeur des humidités relatives moyennes annuelles est de $82 \%$ pour les valeurs maximales et $57 \%$ pour les valeurs minimales.

Bargny fait partie d'un grand ensemble surnommé Plateau de Bargny qui forme un plan incliné vers le Sud, où il se termine par un talus d'une dizaine de mètres de dénivellation, érodé par les rivières et les avancées de la mer. La région se caractérise par une présence importante de marne feuilletée à attapulgite qui s'est transformée progressivement en un matériau d'abord grumeleux, granuleux, poreux, puis friable (Diop, 2002). Ce type de transformation s'accompagne d'une individualisation de la montmorillonite avec la calcite d'où le caractère gonflant des sols. Au niveau des pentes, il s'est développé des sols souvent caillouteux, très riches en Ca et Mg (Diop, 2002). La végétation est essentiellement composée d'arbustes épineux, de quelques baobabs (Adansonia digitata) et figuiers (Ficus caricas).

\section{Dispositif expérimental}

La plantation du matériel végétal de l'étude, composé de deux provenances (facteur 1 : Casamance et Diobass), a été réalisée en 2008. Elle a été effectuée dans de grands potets d'un mètre de côté disposés en lignes, avec des plants élevés en pépinière ( 3 mois), à la densité de 625 plants à l'hectare $(4 \mathrm{~m} \times 4 \mathrm{~m})$. Un travail du sol (facteur 2) qui a consisté en un sous-solage croisé une dent a été effectué à $40 \mathrm{~cm}$ de profondeur sur une partie de la parcelle avant la plantation alors que le reste (témoin) de la parcelle n'a fait l'objet $\mathrm{d}^{\prime}$ aucune préparation de terrain.

Après plantation, les plants de JCL ont été irrigués durant 12 mois, à raison de deux arrosages de $8 \mathrm{~L} /$ plant/semaine pour leur assurer une bonne alimentation hydrique. En première année, chaque plant a ainsi reçu l'équivalent de $64 \mathrm{~L}$ d'eau/mois, soit un cumul annuel de $768 \mathrm{~mm}$ équivalent précipitations (en plus des $378 \mathrm{~mm}$ de pluies reçus) qui se situe plus en conditions soudano-guinéennes que sahéliennes.

La parcelle élémentaire était constituée de 10 plants choisis au hasard et étiquetés dans un carré de $36 \mathrm{~m}$ de côté contenant 100 plants de JCL. Ces parcelles ont été délimitées à l'intérieur des plantations pour éviter les effets de bordure (figure 1).

Le dispositif expérimental en split-block comprenait trois blocs et deux facteurs étudiés avec deux niveaux chacun : Provenances (Diobass et Casamance randomisés au niveau de chaque bloc dans deux parcelles principales) et

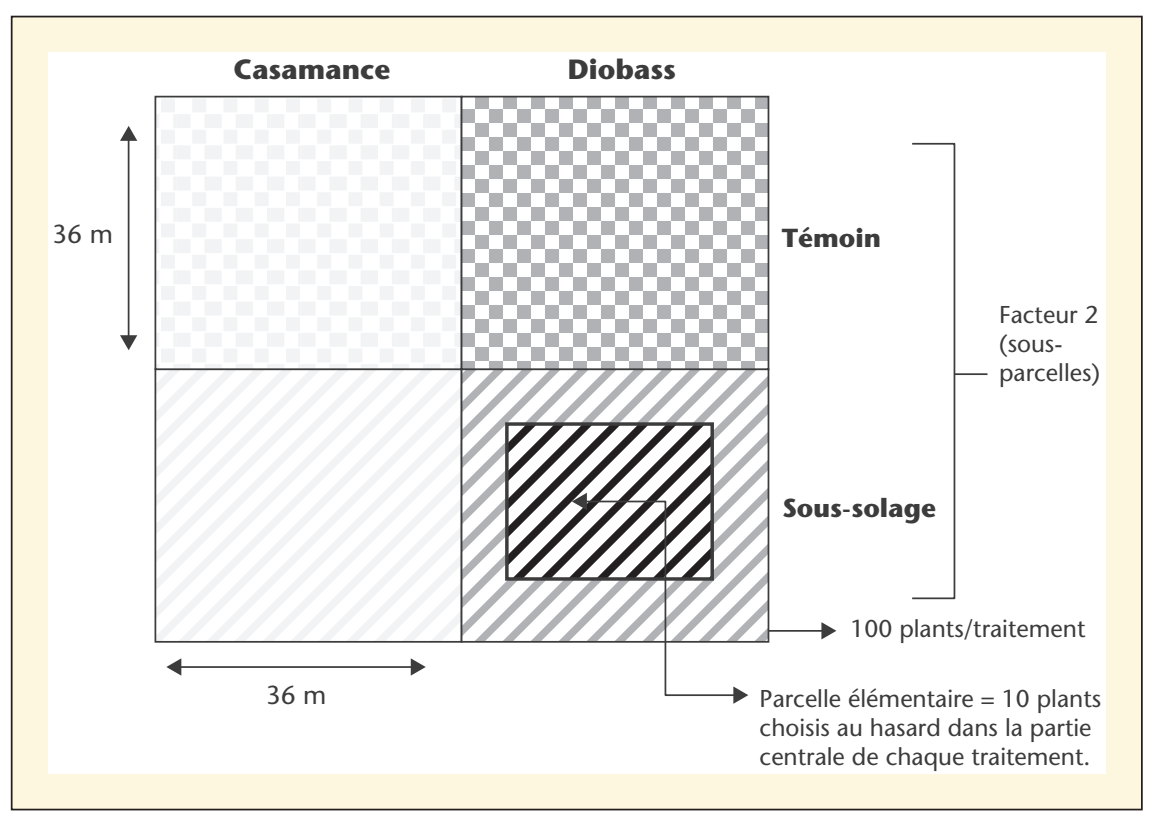

Figure 1. Représentation d'un des trois blocs du dispositif expérimental en split-block comprenant deux facteurs à deux niveaux chacun : Provenance (Casamance et Diobass) en parcelles principales et Travail du sol (Sous-solage et Témoin) en sous parcelles. 
Travail du sol (sous-solage et témoin, randomisés également au niveau de chaque bloc et non au niveau de chaque parcelle principale, dans deux parcelles secondaires).

Les observations ont été effectuées sur les plants de JCL âgés de 24 mois, sur les variables suivantes: le taux de survie déterminé comme suit :

$$
\mathrm{T}=\frac{x}{N t} \times 100
$$

où $N_{\mathrm{t}}$ représente le nombre total des plants et $x$ le nombre des plants survivants; le diamètre au collet de la tige principale; le nombre de ramifications primaires basses (branches de premier ordre situées à une hauteur inférieure ou égale à $15 \mathrm{~cm}$ du sol); le nombre de ramifications primaires hautes (branches de premier ordre situées à une hauteur supérieur à $15 \mathrm{~cm}$ du sol) ; le nombre total de ramifications primaires; le nombre total de ramifications secondaires (nombre total de branches de second ordre); la hauteur totale (longueur du plus long brin) ; le diamètre moyen du houppier $\left(d_{1}=\right.$ suivant la direction parallèle à la ligne de plantation et $d_{2}=$ suivant la direction perpendiculaire à la ligne de plantation); le nombre de ramifications primaires fructifères; le nombre de ramifications secondaires fructifères; le taux de ramifications secondaires fructifères :

Taux $=\frac{\text { nombre ramifications secondaires fructifères }}{\text { nombre de ramifications secondaires }} \times 100$;

le nombre total de branches fructifères ; le nombre total de fruits et la masse unitaire des graines mûres.
Les mesures de longueurs ont été effectuées avec un ruban gradué en mètre sauf pour la hauteur qui a été mesurée à l'aide d'une perche emboitée de mensuration $(8 \mathrm{~m})$. Les mesures de masse ont été obtenues avec une balance de précision 1/100. Toutes les données recueillies ont été saisies sur Excel $^{\mathrm{TM}}$ et soumises à une analyse de variance (ANOVA), suivie d'un test de comparaison de moyennes de Duncan pour identifier les traitements significativement différents. Les analyses ont été effectuées avec le logiciel SAS.

\section{Résultats}

\section{Croissance de Jatropha curcas L.}

Diamètre au collet de la tige principale Après deux ans, la croissance du diamètre au collet de la tige principale de la provenance JCL Casamance $(11,4 \mathrm{~cm} \pm$ $0,2)$ a significativement $(p=0,0351$; tableau 1) été supérieure à celle de la provenance Diobass $(9 \mathrm{~cm} \pm 0,2)$. Par ailleurs, le sous-solage a semblé augmenter le diamètre des deux provenances (Casamance : $12 \pm 1 \mathrm{~cm}$ dans la parcelle sous solée et $11 \pm 0,8 \mathrm{~cm}$ dans le témoin ; Diobass : $10 \pm 0,7 \mathrm{~cm}$ pour le travail du sol et $8 \pm 0,6 \mathrm{~cm}$ pour le témoin).

\section{Diamètre moyen du houppier (DH)}

Le diamètre moyen du houppier (DH) de la provenance Casamance $(3,2 \mathrm{~m} \pm$ $0,2)$ a significativement $(p=0,0297$; tableau 1) été supérieur à celui de la provenance Diobass $(2,3 \mathrm{~m} \pm 0,2)$. DH avait également tendance à augmenter avec le travail du sol (Casamance : 3,3 $\pm 0,3 \mathrm{~m}$ dans la parcelle préparée et $3,1 \pm 0,3 \mathrm{~m}$ dans la parcelle-témoin ; Diobass : 2,5 $\pm 0,1 \mathrm{~m}$ dans la parcelle travaillée et $2,2 \pm 0,2 \mathrm{~m}$ dans la parcelle-témoin).

\section{Hauteur totale}

La hauteur totale des plants de JCL a également significativement ( $\mathrm{p}=$ 0,0012 ; tableau 1) varié en fonction de la provenance, avec une moyenne de $1,8 \mathrm{~m}( \pm 0,004)$ pour la provenance Casamance et $1,5 \mathrm{~m}( \pm 0,004)$ pour la provenance Diobass. Par contre, le soussolage n'a pas eu d'effet significatif sur cette variable $(p=0,3482)$.

\section{Taux de survie des plants de Jatropha curcas L.}

La survie des plants a semblé être meilleure dans la parcelle travaillée (99\%) que dans le témoin (92\%) chez la provenance Diobass. Par contre elle est restée invariable (97\%) chez la provenance Casamance, quel que soit le traitement.

\section{Architecture du port des plants de Jatropha curcas L}

Ramifications primaires basses (RPB) et hautes (RPH)

Le nombre de RPB (hauteur $\leq 15 \mathrm{~cm}$ ) des plants de JCL a significativement varié avec la provenance $(p=0,0022$; tableau 2) : 3,0 $\pm 0,1$ pour la provenance Casamance et $2 \pm 0,1$ pour la provenance Diobass. Le travail du sol a également fait varier le nombre de RPB $(p=0,0192$; tableau 2) : $3 \pm 0,1$ pour la parcelle travaillée et $2,1 \pm 0,1$ pour la parcelle témoin.

Par contre, la provenance $(p=0,8511)$, le sous-solage $(p=0,1460)$ et l'interaction entre les deux facteurs $(p=0,1935$; tableau 2) n'ont pas eu d'effets significatifs sur le nombre de RPH (hauteur $>$ $15 \mathrm{~cm}$ ). Par conséquent, le nombre

Tableau 1. Effet du travail du sol (sous-solage) et de la provenance (Prov) sur les variables : diamètre au collet de la tige principale (DCTP), diamètre du houppier $(D H)$, hauteur totale $(H)$ et taux de survie de plants de Jatropha curcas âgés de 24 mois, plantés en conditions irriguées sur le site de la SOCOCIM (Bargny, Sénégal).

\begin{tabular}{|c|c|c|c|c|c|c|c|c|c|c|c|}
\hline \multirow[t]{2}{*}{ Variables } & \multicolumn{2}{|c|}{ Provenances (Prov) } & \multirow[t]{2}{*}{$\mathbf{P}>\mathbf{F}$} & \multicolumn{2}{|c|}{ Travail du sol (TDS) } & \multirow[t]{2}{*}{$\mathbf{P}>\mathbf{F}$} & \multicolumn{4}{|c|}{ Interaction Prov $\times$ TDS } & \multirow[t]{2}{*}{$\mathbf{P}>\mathbf{F}$} \\
\hline & Casa & Diob & & $\begin{array}{l}\text { Sous-solage } \\
\text { (SS) }\end{array}$ & $\begin{array}{l}\text { Témoin } \\
\text { (Tem) }\end{array}$ & & $\begin{array}{l}\text { Casa } \times \\
\text { ss }\end{array}$ & $\begin{array}{l}\text { Diob } \times \\
\text { SS }\end{array}$ & $\begin{array}{l}\text { Casa } \times \\
\text { Tem }\end{array}$ & $\begin{array}{l}\text { Diob } \times \\
\text { Tem }\end{array}$ & \\
\hline DCTP & $11,4 \pm 0,2$ & $9 \pm 0,2$ & $0,0351^{*}$ & $10,7 \pm 0,02$ & $9,4 \pm 0,002$ & 0,1855 & $12 \pm 1$ & $10 \pm 0,6$ & $11 \pm 0,8$ & $8 \pm 0,7$ & 0,6921 \\
\hline $\mathrm{DH}$ & $3,2 \pm 0,2$ & $2,3 \pm 0,2$ & $0,0297^{*}$ & $2,9 \pm 0,2$ & $2,6 \pm 0,2$ & 0,3317 & $3,3 \pm 0,3$ & $2,5 \pm 0,1$ & $3,1 \pm 0,3$ & $2,2 \pm 0,2$ & 0,8302 \\
\hline $\mathrm{H}$ & $1,8 \pm 0,004$ & $1,5 \pm 0,004$ & $0,0012^{*}$ & $1,7 \pm 0,004$ & $1,7 \pm 0,004$ & 0,3482 & $2 \pm 0,05$ & $1,5 \pm 0,06$ & $2 \pm 0,03$ & $1,5 \pm 0,05$ & 0,1395 \\
\hline $\begin{array}{l}\text { Taux } \\
\text { de survie }\end{array}$ & $97 \pm 9,25$ & $95 \pm 9,25$ & 0,4411 & $98 \pm 9,25$ & $94 \pm 9,25$ & 0,1170 & $97 \pm 1$ & $99 \pm 1$ & $97 \pm 1$ & $92 \pm 3,05$ & 0,1170 \\
\hline
\end{tabular}

Casamance $=$ Casa $;$ Diobass $=$ Diob $;$ Témoin $=$ Tem ; ${ }^{*}$ significatif au seuil de 0,05. 
Tableau 2. Effet du travail du sol (sous-solage et témoin) et de la provenance (Casamance et Diobass) sur le nombre de ramifications primaires basses $(R P B)$, le nombre de ramifications primaires hautes $(R P H)$, le nombre total de ramifications primaires (RPT), le nombre de ramifications secondaires (RS), le nombre de ramifications primaires fructiferes (RPF), le nombre de ramifications secondaires fructiferes (RSF), le taux de ramifications secondaires fructiferes (TRSF) et le nombre total de branches fructiferes (BFT) d'une plantation irriguée de Jatropha curcas L. âgé de 24 mois, localisée dans le site de la SOCOCIM (Bargny, Sénégal).

\begin{tabular}{|c|c|c|c|c|c|c|}
\hline \multirow[t]{2}{*}{ Variables } & \multicolumn{2}{|c|}{ Provenances } & \multirow[t]{2}{*}{$\mathbf{P}>\mathbf{F}$} & \multicolumn{2}{|c|}{ Sous-solage } & \multirow[t]{2}{*}{$\mathbf{P}>\mathbf{F}$} \\
\hline & Casa & Diob & & SS & Tem & \\
\hline RPB & $3 \pm 0,1$ & $2 \pm 0,1$ & $0,0022^{*}$ & $3 \pm 0,1$ & $2,1 \pm 0,1$ & $0,0192^{*}$ \\
\hline $\mathrm{RPH}$ & $5 \pm 0,5$ & $5 \pm 0,5$ & 0,8511 & $4,3 \pm 0,5$ & $5 \pm 0,5$ & 0,3009 \\
\hline RPT & $8 \pm 0,6$ & $7 \pm 0,6$ & $0,0516^{*}$ & $7 \pm 0,6$ & $7,2 \pm 0,6$ & 0,7454 \\
\hline RS & $18,5 \pm 2,5$ & $8 \pm 2,5$ & $0,0213^{*}$ & $15,1 \pm 2,5$ & $11,2 \pm 2,5$ & 0,2513 \\
\hline RPF & $2,6 \pm 0,10$ & $1,2 \pm 0,10$ & $0,0023^{*}$ & $1,5 \pm 0,10$ & $2,2 \pm 0,10$ & $0,0234^{*}$ \\
\hline RSF & $4,3 \pm 0,7$ & $1 \pm 0,7$ & $0,0021^{*}$ & $2,6 \pm 1,0$ & $2,4 \pm 1,0$ & 0,7569 \\
\hline TRSF & $23,2 \pm 1,7$ & $12,5 \pm 1,7$ & $0,0021^{*}$ & $17,2 \pm 1,7$ & $19,4 \pm 1,7$ & 0,2513 \\
\hline BFT & $7 \pm 1,2$ & $2 \pm 1,2$ & $0,0014^{*}$ & $4,1 \pm 1,2$ & $5 \pm 1,2$ & 0,4336 \\
\hline
\end{tabular}

*significatif au seuil de 0,05 .

total (RPT) de ramifications primaires $(\mathrm{RPB}+\mathrm{RPH})$ a seulement varié en fonction $d u$ facteur provenance ( $p=$ 0,0516; tableau 2).

\section{Ramifications secondaires (RS) de Jatropha curcas}

Le nombre de RS a varié suivant la provenance $(p=0,0213$; tableau 2). La provenance Casamance a ainsi produit plus de RS $(18,5 \pm 2,5)$ que la provenance Diobass $(8 \pm 2,5)$. Le nombre de RS a semblé également augmenter sous l'effet du sous-solage chez la provenance Casamance avec 20,2 \pm 3 RS pour la partie sous solée et $17 \pm 3,6 \mathrm{RS}$ pour le témoin contre $10 \pm 2,4$ RS et $6 \pm$ $1,3 \mathrm{RS}$, respectivement chez la provenance Diobass.

\section{Ramifications primaires fructiferes (RPF) de Jatropha curcas}

La fructification a commencé en même temps chez les deux provenances, à partir du quatorzième mois après plantation. Elle a été continue depuis cette date. Le nombre de ramifications primaires fructifères (RPF) a été plus élevé $(2,6 \pm 0,1)$ pour la provenance Casamance que pour la provenance Diobass $(1,2 \pm 0,1$ RPF ; $p=0,0023$; tableau 2). $D^{\prime}$ autre part, le travail du sol a eu une influence sur le nombre de RPF ( $p=$ 0,0234 ; tableau 2) : 1,5 $\pm 0,1$ RPF pour la parcelle sous solée et 2,2 $\pm 0,10 \mathrm{RPF}$ pour le témoin.

Pour les ramifications secondaires fructifères (RSF), seul l'effet de la provenance a été significatif sur cette variable $(p=0,0021)$ avec des valeurs moyennes quatre fois plus élevées $(4,3 \pm 0,8)$ pour la provenance Casamance que pour Diobass $(1 \pm 0,8)$. Le taux de RSF a également suivi la même tendance que le nombre de RSF (tableau 2), avec un effet "provenance " significatif $(p=0,0021)$ qui s'est manifesté par une production de ramifications secondaire fructifère presque deux fois plus importante chez la provenance Casamance
$(23,2 \pm 1,7 \%)$ que chez la provenance Diobass (12,5 $\pm 1,7 \%)$.

Par conséquent, le nombre total de branches fructifères a été plus élevé ( $p=0,0014$; tableau 2) chez la provenance Casamance $(7 \pm 1,2)$ que chez que la provenance Diobass $(2 \pm 1,2)$.

\section{Production de fruits et de graines}

La provenance Casamance avec en moyenne $11 \pm 0,9$ fruits par arbre a produit trois fois plus de fruits ( $p=$ 0,0153 ; tableau 3) que la provenance Diobass $(4 \pm 0,9)$. Par ailleurs, après deux ans de plantation, les facteurs "sous-solage", "provenance", de même que l'interaction des deux facteurs n'ont révélé aucun effet significatif sur le nombre des graines par fruit mûr (respectivement $p=0,4259 ; p=$ 0,3185 et $p=0,3688$ ) et sur la masse unitaire des graines (respectivement $p=0,0991 ; p=0,8842$ et $p=0,3773)$.

Tableau 3. Effet du travail du sol (sous-solage et témoin) et de la provenance (Casamance et Diobass) sur le nombre total de fruits (NFT) et la masse unitaire de graines mûres (MUGM) dans une plantation irriguée de Jatropha curcas âgée de 24 mois localisée dans le site de la SOCOCIM (Bargny, Sénégal).

\begin{tabular}{|c|c|c|c|c|c|c|c|c|c|c|c|}
\hline \multirow[t]{2}{*}{ Variables } & \multicolumn{2}{|c|}{ Provenances } & \multirow[t]{2}{*}{$\mathbf{P}>\mathbf{F}$} & \multicolumn{2}{|l|}{ Sous-solage } & \multirow[t]{2}{*}{$\mathbf{P}>\mathbf{F}$} & \multicolumn{4}{|c|}{ Interaction Provenance x Sous-solage } & \multirow[t]{2}{*}{$\mathbf{P}>\mathbf{F}$} \\
\hline & Casa & Diob & & $\begin{array}{l}\text { Sous-solage } \\
\text { (SS) }\end{array}$ & $\begin{array}{l}\text { Témoin } \\
\text { (Tem) }\end{array}$ & & Casa - SS & Diob - SS & Casa - Tem & Diob - Tem & \\
\hline NFT & $11 \pm 0,9$ & $4 \pm 0,9$ & $0,0153^{*}$ & $6 \pm 0,8$ & $9 \pm 0,8$ & 0,1800 & $9,4 \pm 3$ & $2,5 \pm 1,3$ & $12,2 \pm 1$ & $5,3 \pm 1,1$ & 0,9926 \\
\hline MUGM & $0,2 \pm 0,01$ & $\begin{array}{l}0,2 \pm \\
0,01\end{array}$ & 0,8842 & $\begin{array}{l}0,11 \pm \\
0,007\end{array}$ & $\begin{array}{l}0,21 \pm \\
0,007\end{array}$ & 0,0991 & $0,1 \pm 0,03$ & $\begin{array}{l}0,09 \pm \\
0,02\end{array}$ & $0,2 \pm 0,02$ & $0,2 \pm 0,1$ & 0,3773 \\
\hline
\end{tabular}

Casa $=$ Provenance Casamance $;$ Diob $=$ Provenance Diobass ; ${ }^{*}$ significatif au seuil de $0,05$. 


\section{Discussion}

Le travail du sol n'a pas influencé les variables de croissance (diamètre au collet de la tige principale, diamètre du houppier et hauteur totale), I'architecture du port aérien (nombre de ramifications primaires, de ramifications secondaires et de ramifications fructifères), le nombre de fruits et la masse des graines. Par contre, il a eu un effet significatif sur le nombre de ramifications primaires basses et le nombre de ramifications primaires fructifères. Par contre, Huwe (2003) avait noté que le travail du sol influence à la fois les processus biotiques et abiotiques, la modification des propriétés structurelles telles que les fissures, les agrégats et la continuité des pores, ainsi que l'aération des sols, les niveaux de température et I'humidité, ce qui favorise la croissance des végétaux. Pour Jatropha curcas, cela n'a pas été confirmé probablement parce que l'espèce a confirmé son aptitude à bien s'accommoder de la plupart des conditions édaphiques (Dommergue et Pirot, 2008). Elle serait même, selon Godin et Spensley (1971), capable de croître entre les rochers sous lesquels il y a un peu de terre et pourrait même être cultivée sur des sols secs et caillouteux. Il faut également noter que les plants de JCL ont été plantés dans de grands potets ameublis, ce qui devrait améliorer les conditions $d$ 'alimentation hydrique et nutritionnelle des plants et de croissance des racines sur l'ensemble de l'essai.

Cependant, la croissance de l'espèce a significativement été fonction de la provenance, notamment en ce qui concerne le diamètre au collet de la tige principale, la hauteur totale et le diamètre du houppier. Pour toutes ces variables de croissance, la provenance Casamance a enregistré les valeurs les plus élevées.

Le taux de survie de JCL n'a pas varié significativement sous l'effet des facteurs sous-solage et provenance, de même que sous l'effet de l'interaction entre ces deux facteurs. Les taux de survie ont varié entre 92 et $99 \%$ pour les deux provenances. Des valeurs similaires ont été observées par Samba et al. (2007) dans la station de Ndiol (Saint-Louis, Sénégal) avec des taux de survie de 99,4\% pour des plants issus de semis de graines fraîchement récoltées. Ces résultats sont meilleurs que ceux de Heller (1992) au Sénégal qui a observé des taux plus bas (5 et $50 \%$ ) avec le semis direct. II importe cependant de rappeler que les plants de notre étude et ceux de Samba et al. (2007) étaient en conditions irriguées. Pour l'architecture du port aérien, la provenance a eu un effet (i) significatif sur le nombre de ramifications secondaires (ii) et non significatif sur le nombre de ramifications primaires. La provenance Casamance présente une ramification secondaire plus importante $(18,5)$ que celle de la provenance Diobass (8). Elle présente un taux de ramifications secondaires fructifères $(23,24 \pm 1,65 \%)$ deux fois plus élevé que celui de Diobass $(12,5 \pm 1,65 \%)$ et un nombre total de branches fructifères (7) 3,5 fois supérieur à celui de Diobass (2). En plus de l'effet de la provenance, le sous-solage a fait varier le nombre de ramifications primaires.

Les résultats sur I'architecture du port aérien sont en harmonie avec ceux de Dommergue et Pirot (2008) qui ont noté que la préparation du sol entraîne un développement plus rapide de la plante, avec une cime large (plusieurs mètres de haut avec des ramifications). De même, Kouyaté et al. (2007) rapportent que le travail du sol influence directement le développement des cultures. Cet état de fait sous-entend que plus les plants sont ramifiés plus la production devient importante.

Ces résultats soutenus par Domergue et Pirot (2008), stipule qu'une ramification précoce de la plante aurait un effet déterminant sur le rendement car les inflorescences se développent seulement au bout des branches donc plus la plante a de branches plus elle produira de fruits. En plus du nombre de ramifications élevé, la provenance Casamance produit plus de fruits (11 fruits/arbre) que la provenance Diobass (4 fruits/arbre). Sous irrigation depuis le début, la plantation a été en fructification continue depuis le quatorzième mois de plantation. En effet Jongschaap et al. (2007) ont également confirmé que la disponibilité de l'eau prolonge la durée de floraison.

Des essais provenances sur des accessions locales de JCL menés par divers auteurs en Inde (Basha et Sujatha, 2007 ; Kaushik et al., 2007 ; Patolia et al., 2007b) ont révélé que la base génétique des provenances indiennes est assez réduite (Basha et Sujatha,
2007) et que des différences significatives dans la morphologie des plants (hauteur, indice de surface foliaire, diamètre au collet, nombre de branches primaires, secondaires et tertiaires) et sur les variables de rendement (nombre de fruits/plant, nombre de graines/ plant, la masse des graines et la teneur en huile) ont été observées entre les provenances (Patolia et al., 2007). Dans notre étude, la provenance et le soussolage, de même que l'interaction des facteurs n'ont pas eu d'influence sur le nombre de graines/fruit ni sur leur masse unitaire. Au Nicaragua où 1200 ha avaient été plantés avec des variétés locales et des variétés du Cap Vert, la variété locale avait présenté, selon Domergue et Pirot (2008) des graines plus grosses mais la variété du Cap Vert avait un meilleur rendement.

En conclusion de cette étude on peut dire que chez Jatropha curcas I'influence de la provenance s'est manifestée sur les variables de croissance, I'architecture de l'arbre et sur le nombre de fruits. En général, les résultats obtenus ont été meilleurs chez la provenance Casamance.

Par ailleurs, le sous-solage a tendance à améliorer la croissance de l'espèce (diamètres au collet de la tige principale et diamètre du houppier) et la ramification (nombre de ramifications primaires basses, nombre de ramifications fructifères primaires et nombre de ramifications secondaires).

Tout en admettant la complexité de I'influence du sous-solage sur les plants de Jatropha curcas, on peut considérer comme Chehaibi et al. (2008), que le travail du sol a un effet favorable sur le développement des plants. II améliore en particulier la vitesse de croissance.

Chez la provenance Casamance, le nombre de fruits est supérieur à 18 fruits/arbre/an. Cependant, concernant le nombre de fruits et la masse des graines, les études doivent être poursuivies. C'est en effet la connaissance du comportement des provenances dans leur environnement et de leur plasticité face aux contraintes du milieu qui faciliteront le choix des taxons les plus appropriés et les techniques à mettre en œuvre, pour un bon développement et une meilleure productivité de Jatropha curcas.

Cette étude a mis en évidence I'influence de la provenance et de la 
préparation du sol sur les plants de Jatropha curcas dans la zone considérée. Il est important de poursuivre les études pour évaluer la masse des fruits et des graines/unité de surface/an et de déterminer la qualité et la quantité de I'huile des graines en fonction de la provenance et du travail du sol.

\section{Liens d'intérêts}

aucun

\section{RÉFÉRENCES}

Basha SD, Sujatha M. Inter and intrapopulation variability of Jatropha curcas (L.) characterized by RAPD and ISSR markers and development of population-specific SCAR markers. Euphytica 2007 ; 156 : 375-86.

Chehaibi S, Hannachi C, Pieters JG, Verschoore RA. Effets des outils de reprise de labour sur l'état structural du sol et le rendement d'une culture de pomme de terre. Tropicultura 2008 ; 26 : 93-7.

Diallo A, Diakhaté MA, Bâ A, Diarra B, Sow M. Profil de développement durable de la commune de Bargny. Agenda 21 de la commune de Bargny, 2006.

Diop M. Conception de structures routières sur marnes et argiles gonflantes. Etude de cas : Bargny-Diamniadio. Projet de fin d'études en vue de l'obtention du diplôme d'ingénieur de conception. Université Cheikh Anta Diop de Dakar, Ecole Supérieure Polytechnique, Département de Génie Civil, 80 pages + Annexes, 2002.

Dommergue M, Pirot R. Jatropha curcas L. : rapport de synthèse bibliographique CIRAD.
Avenue d'Agropolis 34398 Montpellier Cedex 5 AGROgeneration, 45-47 rue de Monceau 75008 Paris, 2008.

Emesto SE. Biofertilizers for Jatropha curcas L. (Euphorbiaceae) Grown in Different Planting Media. International Conference on Environmental Research and Technology, ICERT 2008. http://www.scribd.com/doc/45310464/ Bio-Fertilizers-for-Jatropha-Curcas-L-Euphor biaceae-Grown-in-Different-Planting-Media.

Godin V, Spensley P. Oils and Oilseeds. TPJ Crop and Product Digest $1971 ; 1$ : 107-10.

Gübitz GM, Mittelbach M, Trabi M. Exploitation of tropical oil seed plant Jatropha curcas L. Bioresource Tehnology 1999 ; 67 : 77-82.

Heller J. Studies on genotypic characteristic and propagation and cultivation method for physic nuts (Jatropha curcas L.). Dr. Kovac, Hamburg, 1992.

Heller J. Physic nut. Jatropha curcas L. In: International Plant Genetic Resources Institute (IPGRI), Promoting the conservation and use of underutilized and neglected crops. Prom Underused Crops 1, 1996 : 1-66.

Huwe $B$. The role of soil tillage for soil structure. In: A El Titi (Ed.), Soil tillage in agroecosystems, 2003. CRC, Boca Raton, Florida : 27-49.

Jones $\mathrm{N}$, Miller JH. Jatropha curcas. A multipurpose species for problematic sites. L.r. series, Editor. World Bank, 1992.

Jongschaap REE, Corré WJ, Bindraban PS, Brandenburg WA. Claims and Facts on Jatropha curcas L. Global Jatropha curcas evaluation, breeding and propagation programme. Plant Research International B.V., Wageningen Stichting Het Groene Woudt, Laren, October 2007 Report 158.
Kaushik N, Kumar K, Kumar S, Kaushik N, Roy $\mathrm{S}$. Genetic variability and divergence studies in seed traits and oil content of Jatropha (Jatropha curcas L.) accessions. Biomass and Bioenergy 2007 ; 31 : 497-502.

Kouyaté AM, Van Damme P, Goyens S, De Neve S, Hofman G. Evaluation de la fertilité des sols à Detarium microcarpum Guill. \&Perr. Tropicultura $2007 ; 25$ : 65-9.

Legendre B, Mergeai G, Terren M. Etablissement de plantations de Jatropha. Note agronomique, n³, 2009.

Makkar HPS, Becker K. Jatropha curcas toxicité : l'identification de principe toxique (s). Procédures internationales, $5^{e}$ symposium sur les plantes toxiques, 1997. San Angelo, Texas, USA, mai 19-23.

Patolia JS, Chikara J, Prakash AR, Ghosh A, Chaudharry DR, Bhuva HM. Provenance trials for selection of high yielding Jatropha curcas on wastelands. Expert seminar on Jatropha curcas L. Agronomy and genetics. 26-28 March 2007, Wageningen, the Netherlands, Published by FACT Foundation.

Rijssenbeek WHR, Jongschaap R, Lutzeyer HJ, Venturi P. Expert Meeting Jatropha, Brussels, 2007.

Samba ANS, Diallo B, Diop M, et al. Jatropha curcas: seed germination and propagation methods, 2007. http://www.jatrophaalliance.org/fileadmin/documents/knowledgepool/SambaDiallo_Jatropha_Seed_Germination_ and_Propagation.pdf

USDA ANGRPNGRLBM. Jatropha curcas infromation from Germplasm Resources Information Network - (GRIN) [Online Database]. http://www.ars-grin.gov/cgi-bin/npgs/html/ taxon.pl?20692. Nov. 9, 2007. 